\title{
Quand les acteurs mettent en mots leur expérience
}

\author{
Sylvie Guignon, doctorante
}

Université Laval

Joëlle Morrissette, doctorante

Université Laval

\begin{abstract}
Résumé
Cet article offre une réflexion sur la place des acteurs dans la recherche en s'appuyant sur une investigation qui a porté sur des interventions de coaching en entreprise. Il a pour but de montrer comment les choix qui ont présidé à cette recherche témoignent d'une conception d'acteur compétent, c'est-à-dire capable de réfléchir à ses pratiques et capable d'en parler. Ainsi sont présentées les propositions théoriques qui ont permis d'éclairer l'agir professionnel des coachs, la méthode d'enquête qui a conduit à la construction de récits de pratique ancrés dans leur expérience, de même que la méthode d'analyse inductive qui a facilité la mise en relief des savoirs pratiques mobilisés par des coachs lors d'une intervention. Finalement, une réflexion critique sur ces différentes étapes, en relation avec le statut des données et les places respectives de l'acteur et du chercheur sera posée.
\end{abstract}

Mots-clés : ACTEUR COMPÉTENT, SAVOIRS PRATIQUES, AGIR PROFESSIONNEL, RÉCIT DE PRATIQUE, APPROCHE INDUCTIVE

\section{Introduction}

La recherche adopte traditionnellement une approche de type research down dans nombre de domaines tels que ceux de la formation enseignante, de la didactique, ou encore de la gestion (par exemple, dans le cadre de l'application de modélisation d'optimisation de flux). Souvent réalisée dans la perspective d'une démarche déductive, elle s'alimente de règles théoriques déjà posées et vise à en vérifier la bonne application. Cette perspective, plutôt prescriptive, prend appui sur des conceptualisations théoriques qui sont mises en relation avec l'agir des acteurs étudiés, amenant souvent à pointer des manques, des écarts. Malgré les apports significatifs de

RECHERCHES QuALITATIVES - Vol .26(2), 2006, pp. 19-38.

ISSN 1715-8705 - http://www.recherche-qualitative.qc.ca/Revue.html

(C) 2007 Association pour la recherche qualitative 
cette approche pour notre compréhension collective de différents phénomènes, il n'en demeure pas moins que cette optique fait peu de cas du point de vue des acteurs, considérant leur vision comme un cas particulier, alors qu'elle pourrait être envisagée comme un point de vue complémentaire unique. Rappelons d'ailleurs que Blumer (1937), et d'autres chercheurs issus de la tradition sociologique de Chicago à sa suite, ont été parmi les premiers à reconnaître la compétence des acteurs en introduisant différentes méthodes de recherche qui mettent à l'avant-scène le sens que les acteurs donnent aux situations qu'ils vivent, telles que l'observation participante ou encore le courrier privé. La perspective de recherche développée par cette tradition, connue sous l'appellation «interactionnisme symbolique », est aujourd'hui reconnue par plusieurs auteurs (par exemple, Pessin, 2004) qui considèrent qu'elle a renouvelé de façon très féconde la compréhension des problématiques urbaines, des mondes du travail, de la culture et de l'art, et tracé ainsi la voie à de nombreuses recherches contemporaines dans différents domaines.

Dans le cadre d'une recherche portant sur la pratique du coaching en entreprise, menée par l'une d'entre nous (Guignon, 2006), nous avons adopté une perspective selon laquelle l'expression de l'acteur est ainsi valorisée, accordant par là-même une place importante à la compréhension que ce dernier peut avoir de son propre agir. C'est donc à partir du point de vue des coachs que nous nous sommes intéressée aux savoirs pratiques mis en œuvre lors d'une intervention de coaching, à travers certaines dimensions de leur agir professionnel.

Le but de cet article est de montrer comment notre façon de problématiser, d'éclairer cet objet de recherche de même que de l'investiguer suggère une certaine conception de l'acteur, celle d'un acteur compétent au sens où l'entend Giddens (1987), c'est-à-dire capable de réfléchir à son agir et d'en rendre compte lorsque sollicité. L'argumentation ici déployée s'attache donc tout d'abord à rapporter quelques aspects de la problématique traitée par notre recherche en relation avec la place accordée à l'acteur dans les recherches sur le coaching. Nous présentons les choix théoriques retenus qui prennent appui sur des modèles d'acteurs compétents selon lesquels le point de vue de l'acteur sur ses pratiques est considéré unique et pouvant être exprimé lorsque sollicité. Puis, nous relatons l'approche méthodologique choisie pour investiguer les savoirs pratiques des coachs, approche qui s'ancre à des fondements théoriques et méthodologiques en relation avec l'accueil et l'interprétation de la pratique réalisée par l'acteur invité à expliciter son agir. Enfin, nous posons une réflexion critique sur notre perspective qui s'inscrit surtout dans une démarche inductive.

\section{De la place de l'acteur dans les recherches sur le coaching}

Dans le cadre de notre recherche, l'analyse des écrits recensés met en exergue que les auteurs qui étudient le coaching en entreprise adoptent surtout un regard " extérieur ", campant principalement les "décors », c'est-à-dire des cadres d'intervention dans les entreprises (contraintes économiques, évolutions organisationnelles, etc.), des populations cibles (dirigeants, gestionnaires, etc.), des mandats, des démarches, des techniques et des outils mis en œuvre, ou encore établissant des portraits de coachs au 
regard de leur formation, de leur parcours professionnel ou de ce qui est conçu comme leurs "qualités». Ces recherches permettent ainsi d'éclairer le coaching de diverses manières, mais tel que le relève Liljenstrand (2003), elles adoptent un point de vue extérieur en faisant peu de place au sens que l'un des acteurs pivots du processus, le coach lui-même, donne à ses pratiques.

Dès lors, il semble intéressant d'aborder autrement la question du coaching, en privilégiant la parole des acteurs qui le mettent en œuvre, plus précisément celle des coachs, afin d'appréhender avec eux ce qu'ils envisagent de leurs actions durant une intervention en entreprise. Pour ce faire, nous puisons au domaine des savoirs pratiques, domaine de recherche fondé en Amérique du Nord par des auteurs tels que Schön, Elbaz, Connelly et Clandinin, etc. qui ont posé les fondations de ce domaine dans le champ de l'enseignement depuis plus d'une vingtaine d'années, ou encore développé en Europe depuis un peu plus d'une dizaine d'années par des auteurs comme Barbier, Mialaret, Darré ou encore Laborde qui s'ancrent à différents champs professionnels (par exemple, l'agriculture, la kinésithérapie, etc.) pour investiguer les savoirs pratiques. Nous nous appuyons donc sur certaines conceptions de l'acteur qui lui reconnaissent une réflexivité au quotidien, comme celle développée par Schön (1983, 1994, 1996) autour du praticien réflexif ou encore celle de Laborde (1998) autour de l'acteur improvisant. Il convient ici de préciser que le vocable " compétence» ne renvoie pas à une qualité qui s'opposerait par conséquent à " incompétence », mais au sens qu'en donne Giddens (1987) lorsqu'il définit l'acteur social compétent ${ }^{1}$, c'est-à-dire qui jouit d'une marge de manœuvre, dispose de ressources pour agir, réfléchit sur cet agir et peut le théoriser lorsque sollicité. En ce sens, il ne s'agit pas d'une qualité, mais d'une condition d'action.

\section{De la pertinence du point de vue de l'acteur et de sa capacité à l'exprimer}

Le cadre théorique retenu pour éclairer la pratique des coachs en entreprise, à savoir celui de l'acteur compétent tel qu'entendu par Giddens, met tout d'abord l'accent sur la reconnaissance des savoirs pratiques ainsi que sur des éléments permettant d'appréhender l'agir de l'acteur. Il permet aussi d'aborder certains aspects concernant la verbalisation du sens de la pratique et la difficulté que peut rencontrer l'acteur à la produire. À la suite, un dispositif d'aide à la narration visant à faciliter la mise en mots de l'expérience est décrit.

\section{La reconnaissance des savoirs pratiques}

La pertinence accordée au point de vue du praticien passe, entre autres, par la reconnaissance de ses « savoirs pratiques », expression employée surtout en Amérique du Nord, ou "savoirs d'action » en Europe francophone (Barbier, 1996). Plusieurs chercheurs attribuent une « valeur» à ces savoirs issus de l'expérience, construits dans le cadre des différents contextes dans lesquels le praticien œuvre. En effet, des auteurs tels Desgagné (1997, 1998, 2005), qui développe l'axe de la recherche collaborative avec des praticiens enseignants, Schön $(1983,1994,1996)$, qui a exploré la réflexion dans l'action d'un acteur dans une pratique professionnelle, envisagent que ces savoirs pratiques viennent non pas s'opposer aux savoirs théoriques développés par les chercheurs, mais les compléter et étendre la pertinence sociale des modèles proposés. 
Dans cette veine, Cyrulnik (2003), chercheur et neuropsychiatre, soutient qu'il existe un fossé entre chercheur en laboratoire et artisans du terrain: les premiers étudient « un objet partiel, propre, manipulable, vérifiable et réfutable » tandis que les seconds font face à « un objet global, incertain et variable» (p. 72). Ainsi, c'est en posant le constat de la non adéquation des seuls savoirs théoriques face à des situations d'actions professionnelles, autrement dit des contextes incertains et non "fabriqués" en laboratoire, que des auteurs ont avancé l'intérêt de reconnaître l'à-propos des savoirs construits dans l'action, qui se confrontent à la complexité des contextes. Se manifestant dans l'agir du praticien, ils révèleraient une capacité à s'adapter aux situations, à créer des réponses en relation avec le contexte, à mobiliser un « répertoire d'actions » (Schön, 1983) développé et transformé dans l'expérience.

Certains auteurs critiquent le modèle de la rationalité technique qui a dominé le monde de la recherche tout au long du XX ${ }^{\text {ème }}$ siècle. Pour Barbier (1996), chercheur dans le champ des pratiques professionnelles en éducation, ce modèle privilégie des savoirs assimilés aux connaissances théoriques qui sont l'aboutissement de travaux de recherche menés par la communauté dite scientifique, selon des normes de production spécifiques. Ces savoirs sont reconnus socialement et donnent lieu à des modélisations techniques pour utilisation dans les domaines professionnels. Dans ce cadre particulier, la relation entre le monde de la recherche et celui de la pratique s'établit de façon unidirectionnelle et hiérarchisée, le chercheur faisant « don » de sa science au praticien, ce dernier «appliquant» les techniques qui en découlent pour résoudre les problèmes qu'il rencontre dans le cadre de sa pratique.

C'est en des termes semblables que St-Arnaud (1997), qui mène des investigations dans le champ du développement organisationnel en entreprise, parle de ce modèle selon lequel «le savoir guide l'action» et au sein duquel tout le processus de résolution de problème repose sur les outils confiés au praticien et sur ses habiletés à les mobiliser. Pour sa part, Berry (1996), chercheur dans le domaine des sciences de la gestion et du mangement qui s'intéresse à la problématique des échanges entre le monde scientifique et celui de la pratique, illustre par exemple les résultats de l'application de modèles issus de théories dans le cadre du domaine de la gestion. Il retient que ce domaine fait l'objet de recherches scientifiques donnant lieu à la modélisation de certaines de ses pratiques: les modèles financiers, les modèles d'optimisation des flux ou encore les méthodes d'ordonnancement, autant de démarches qui ancrent certains processus de gestion dans les savoirs théoriques. Selon l'auteur, en raison de la complexité des terrains d'application, nombre de ces savoirs théoriques se révèleraient inappropriés. C'est ainsi qu'il note que les modèles scientifiques pourraient rendre le service attendu s'ils étaient employés dans un environnement " aseptisé », où les décisions hiérarchiques seraient prises seulement en fonction de critères "objectifs", mais qu'ils ne sont pas adaptés puisque les contextes de pratique impliquent des processus de négociation dans lesquels interviennent différents enjeux de pouvoir entre acteurs par exemple, des enjeux qui n'entrent pas dans les modélisations théoriques.

Ces différents auteurs considèrent donc que, dans la pratique, les problèmes ne se présentent pas déjà définis tel que le laisse croire le modèle de la rationalité technique, 
c'est-à-dire dépossédés des caractéristiques contextuelles. Ils avancent donc que c'est à ce titre que les savoirs mis en action par l'acteur revêtent un intérêt, puisqu'ils s'organisent en contexte, dans le cadre de situations en partie indéterminées, pour lesquelles les interactions amènent des développements qui peuvent être anticipés ou non. Dès lors, ces savoirs pratiques viennent, non pas s'opposer aux savoirs théoriques pensés par les chercheurs, mais les compléter et étendre la pertinence sociale des modèles proposés en usant des modèles scientifiques pour interroger la situation tout en laissant le libre-arbitre du praticien s'exprimer dans la prise de décision comme le propose Berry (1996), construits à la lumière de l'interprétation de multiples expériences. En ce sens, reconnaître l'à-propos des savoirs pratiques de l'acteur amène à s'intéresser directement à son agir professionnel pour mieux envisager sa compétence.

\section{Une théorie de l'agir professionnel}

Schön $(1983,1994,1996)$ soutient que, en contexte, la pratique professionnelle est caractérisée par l'ambiguïté, la contradiction, l'indétermination, et non par une définition claire et stable d'un problème posé ex nihilo. Ce constat l'amène à supposer l'existence de savoirs pratiques construits dans l'action qui se confrontent à la complexité des situations. En cela, il développe une théorie des savoirs pratiques tacites mobilisés dans l'agir professionnel. Il traite alors d'une prise en compte du contexte de la pratique, d'une réflexion qui préside à l'agir, d'un éventail des actions possibles, ainsi que de pratiques routinières.

\section{Une prise en compte du contexte par le praticien}

Pour Schön (1983, 1994, 1996), le modèle de la rationalité technique néglige des phénomènes qui font partie intégrante de la pratique tels que la complexité, l'incertitude, l'instabilité, la singularité et les conflits de valeurs. Or, il estime que pour construire ses stratégies d'action, le praticien tient compte de ces phénomènes en ce qu'ils sont particuliers à chaque situation rencontrée. On trouve également ces considérations chez Laborde (1998), qui a étudié les savoirs tacites des bertsularis, ces artistes basques qui improvisent durant des joutes des poèmes chantés, versifiés et rimés. Selon cet auteur, les règles de cet art particulier fourniraient à ces artistes des balises sur lesquelles prendre appui pour improviser, et les réactions de l'assistance les amèneraient à créer des agencements inédits. Ainsi, les bertsularis développeraient une capacité à improviser en contexte pour faire face à des situations singulières, compétence qui leur permettrait de prendre en charge les contraintes qui émergent de leur interaction avec les spectateurs au fur et à mesure de l'improvisation.

Ainsi Schön (1983, 1994, 1996) et Laborde (1998) posent que c'est en tenant compte des éléments indéterminés et instables des situations rencontrées que l'acteur définirait les objectifs à poursuivre, les moyens à mettre en œuvre, les décisions à prendre pour atteindre les buts fixés. Celui-ci adapterait donc ses savoirs à une conjoncture particulière en accomplissant un certain type de raisonnement en prenant appui sur les contraintes et les ressources de la situation qui l'amènerait à définir les problèmes auxquels il fait face. 


\section{Des " conversations réflexives " du praticien}

Schön $(1983,1994,1996)$ conçoit en effet que le praticien, confronté à une situation problématique, doit définir le problème qui se pose à lui, apprécier les développements possibles et arrêter une stratégie d'actions en fonction de sa compréhension du contexte particulier de son intervention; ce travail de cadrage lui permettrait de rendre la situation «traitable». Pour l'auteur, ce processus consisterait en une "conversation réflexive » que le praticien engage en lui-même, soit une dialectique entre les savoirs pratiques acquis et l'intervention en cours.

Selon l'auteur, la réflexion, menée en cours d'action et sur l'action (reflexion in action), permettrait de mettre en rapport le problème qui se pose avec les schémas construits dans l'expérience, des théorisations que le praticien dégage de son agir. En fait, ce processus viserait à définir la situation par un jeu d'analogie («voir-comme » ou « faire-comme ») afin de reconnaître les stratégies déjà utilisées dont le praticien pourrait s'inspirer pour agir en situation.

Laborde (1998) rejoint cette conception puisqu'il considère que le bertsulari puiserait à ses expériences pour répondre à la situation en cours, mais surtout projetterait par avance les suites possibles pouvant découler des gestes qu'il pose au fur et à mesure que progresse son improvisation. Bref, les auteurs posent que c'est en menant une réflexion dans le cours de l'action et sur l'action en différé que l'acteur s'adapte et crée une réponse jugée pertinente.

Lorsque Schön (1983, 1994, 1996) et Laborde (1998) évoquent une réflexion posée par l'acteur en cours d'action et sur l'action, ils renvoient aux savoirs pratiques déployés par ce dernier pour composer avec les phénomènes caractérisant les situations qu'il rencontre. Bref, les auteurs posent que c'est en menant une réflexion dans le cours de l'action et sur l'action en différé que l'acteur s'adapte et crée une réponse à partir d'un certain « répertoire d'actions ».

\section{Le " répertoire d'actions " du praticien}

Pour Schön (1983, 1994, 1996), l'activité du praticien serait fondée sur l'usage d'un « répertoire d'actions » qui permettrait d'appréhender et de résoudre les situationsproblèmes auxquelles il est confronté dans sa pratique. L'auteur avance que ce répertoire, en tant qu'éventail de possibles, serait exploité et construit par le biais des différentes «conversations réflexives» qu'engagerait l'acteur avec les situations problématiques qu'il rencontre. Dès lors, il envisage que le praticien serait en mesure de poser des hypothèses en regard de l'ensemble de ses expériences, de « compiler un répertoire d'exemples, de représentations, de compréhensions et d'actions » (p. 175) pour interpréter la situation et agir. Cette délibération du praticien dans l'action, cette dialectique entre expérience et action, lui permettrait donc de comprendre, de projeter et de décider comment adapter les actions connues ou comment en créer de nouvelles pour prendre en compte les spécificités d'une situation. Ce mouvement conversationnel mobiliserait le « répertoire d'actions » et l'enrichirait en retour. Il en résulterait, selon le cas, une validation, un ajustement, un abandon ou un ajout d'une stratégie d'action au «répertoire d'actions» qui serait ainsi enrichi par chaque situation-problème rencontrée. C'est en s'appuyant sur une logique similaire que Laborde (1998) conçoit 
l'existence d'actions répertoriées acquises au cours du temps. En effet, l'auteur estime que l'activité d'un bertsulari permettrait la mise en relation de desseins poursuivis, de contraintes côtoyées, conduisant alors à la création d'une infinité de poèmes chantés improvisés, façonnés par les réactions de la foule avec qui il interagit.

Ainsi pour les auteurs, l'exploitation du répertoire permettrait de caractériser la situation rencontrée, en évaluant en quoi elle est semblable aux situations déjà familières, et en quoi elle s'en différencie, et ce, dans le but d'adapter la réponse à apporter. Les cas connus, familiers, agiraient comme des modèles auxquels seraient comparés chacun des cas présents afin d'en apprécier la spécificité. Dans cette optique, le répertoire tiendrait lieu de collection personnelle de grilles de lecture disponibles pour mieux interpréter les situations rencontrées et donc agir.

\section{Des routines mobilisées par le praticien}

Chaque «conversation réflexive» engagée par l'acteur, tel que décrite par les différents auteurs, contribuerait à enrichir le «répertoire d'actions », autant par confirmation que par création ou restructuration des expériences. Pour Schön (1983, 1994, 1996), c'est ainsi que chacune des situations non familières abordées deviendrait à son tour familière, puisqu'elle finirait par faire partie intégrante des routines d'action du répertoire qui sont disponibles au praticien. Cela renvoie à l'idée que les expériences réussies fixeraient des schémas d'actions pertinents, tandis que les expériences vécues comme des revers ou celles qui surprennent questionneraient les routines, suscitant des modifications, voire leur abolition abandon.

En somme, ces auteurs estiment que l'acteur détient des savoirs pratiques qui se manifestent dans son agir, qui en est la source et l'expression. Tel qu'envisagé, cet agir serait constitué de plusieurs dimensions. De fait, il relèverait d'une capacité à prendre en compte les caractéristiques liées à des contextes singuliers et donc d'une capacité à s'adapter à des situations indéterminées. Également, cet agir se déploierait dans la création de réponses adaptées in situ grâce à une réflexion posée dans le cours de l'action. De plus, il s'appuierait sur un "répertoire d'actions» diversifié qui se développerait et se transformerait dans l'expérience, lequel orienterait la façon de définir les problèmes et d'intervenir pour les résoudre. Enfin, cet agir se dévoilerait au travers de routines qui s'appliquent à la « reconnaissance» des situations, comme au travers de la capacité à créer des solutions innovantes pour faire face aux imprévus des contextes rencontrés. Décrit en ces termes, l'agir de l'acteur pourrait mettre en jeu d'autres caractéristiques que les seuls critères théoriques de la rationalité technique, puisque ce serait la pratique de l'acteur qui permettrait d'engendrer l'action dans des contextes indéterminés et de la reproduire.

La reconnaissance de la compétence de l'acteur amène à s'intéresser au sens qu'il donne à ses actions, et par conséquent, à favoriser l'expression de ce regard porté de l'« intérieur », un point de vue unique sur ses pratiques. Mais, tel que le soulignent Filteau, Bourassa et Leclerc (2001) à propos des savoirs construits dans la pratique, «pour être reconnus et appréciés à leur juste valeur, les savoirs d'expérience doivent pouvoir [...] se rendre visibles et dicibles » (p. 7). Les auteurs soulignent ainsi que l'acteur peut éprouver une certaine difficulté à rendre compte du sens de ses pratiques. 


\section{Une verbalisation du sens de la pratique}

Plusieurs auteurs (par exemple Desgagné, 1994, 2005) s'engagent dans des recherches qui privilégient le point de vue des acteurs pour faire émerger un sens associé aux pratiques. Ils favorisent un regard porté de l' « intérieur » pour questionner les pratiques et, par conséquent, ont en commun une certaine conception des acteurs, centrée sur la reconnaissance de leur compétence. Ils adoptent ainsi une perspective compréhensive qui reconnaît la valeur de la parole des acteurs qui sont en mesure de livrer un sens unique sur les pratiques, un sens à interpréter par le chercheur. Par ailleurs, d'aucuns considèrent que cette expérience peut être difficile à mettre en mots car elle témoigne, entre autres, de savoirs automatisés, implicites et familiers (par exemple, Giddens, 1987; Schön, 1983, 1994, 1996).

Ainsi, pour Giddens (1987), qui propose une conception de l'acteur social compétent, l'acteur ne serait pas forcément en relation consciente avec ses savoirs mis en action. L'auteur marque une différence entre ce qu'il appelle «la conscience discursive » et « la conscience pratique ». Il considère que la distinction entre ces deux consciences s'établit par le discours: l'acteur serait apte à énoncer certaines actions qu'il pose, mais serait incapable d'en verbaliser d'autres. Dans cette perspective, des actions seraient effectuées en connaissance de cause, réfléchies, pesées, jaugées avec des causes et des effets, et a contrario, d'autres seraient plutôt le fruit de routines, d'automatismes, qui ne donneraient plus lieu à réflexion préalable.

De son côté, Schön $(1983,1994,1996)$ considère que le praticien, " même s'il utilise consciemment des théories et des techniques, s'appuie sur des reconnaissances, des jugements et des compétences qui sont tacites » (p. 76). En posant ce postulat, il souligne, tout comme Giddens (1987), que l'acteur ne serait pas en mesure de mettre en mots ses savoirs pratiques dès lors que ceux-ci auraient trait à des actions routinières, des schémas d'actions couramment mis en œuvre. Ainsi, ces auteurs amènent à concevoir que l'acteur évoluerait entre une "compétence consciente », lorsqu'il est attentif à ses propres schémas d'action, et une " compétence inconsciente », lorsqu'il est guidé par une routine dans l'action. Ils soulèvent la difficulté que rencontrerait alors l'acteur pour mettre en mots son expérience lorsque celle-ci renvoie à des automatismes auxquels il n'est plus attentif, obstacle levé s'il a mobilisé des ressources réflexives en cours d'action, par exemple s'il a été surpris d'une quelconque façon par la situation et qu'il a alors dû y être attentif pour résoudre le problème posé.

Or, il appert que certains dispositifs d'expression sauraient atténuer la difficulté posée par la mise en mots de l'expérience. C'est dans cette optique que certains auteurs privilégient la formation (par exemple, Schön, 1983, 1994, 1996), d'autres les entretiens de groupe (par exemple, Filteau et al., 2001), les récits de pratique (par exemple, Desgagné, 2005) ou encore un journal réflexif (par exemple, Bourassa, Serre et Ross, 1999). Ces différentes démarches permettraient d'évoquer des séquences professionnelles, mettant ainsi en relief un « savoir-agir » ou "savoir-intervenir » mis en œuvre en situation. Elles viseraient à aider le praticien à prendre conscience des savoirs cachés dans ses actions, des règles auxquelles il se conforme, des stratégies d'action dont il fait usage. Ainsi, ces différentes démarches représenteraient pour lui des 
occasions de développement professionnel, tout en permettant aux chercheurs de mener des investigations.

\section{Un dispositif d'aide à la narration}

Pour faciliter la mise en mots d'une intervention de coaching par les coachs, nous avons développé un dispositif d'aide à la narration pour notre propre recherche qui prend appui sur les travaux de Bertaux (1997), de Desgagné (2005) et de Desgagné, Gervais et Larouche (2001). L'approche proposée par ces auteurs au travers du récit de pratique nous a semblé pouvoir relever les défis posés par notre investigation, à savoir mettre en relief les savoirs pratiques dont témoigne l'évocation faite par les coachs d'une intervention en entreprise.

Le récit de pratique s'inscrit dans une tradition sociologique qui considère à sa source «l'histoire de vie» d'un acteur. Si «l'histoire de vie» fait référence aux événements qui se sont déroulés, qui ont été vécus par un acteur, il peut sembler fécond d'emprunter « le récit de vie » pour en avoir une meilleure compréhension, étape qui vise une reconstruction des événements par l'entremise des mots. L'acteur témoigne alors de différents sujets liés à sa vie personnelle, Bertaux (1997) dirait qu'il livre son « intériorité ». Le récit de pratique, pour sa part, poursuit d'autres objectifs. Selon Desgagné (2005), il revêt tout son intérêt dès lors que l'attention se porte sur la pratique mise en oeuvre et non sur le « vécu personnel », que la narration d'expériences propres et de leurs contextes d'action est sollicitée. Cette narration se fait donc comme un récit, envisagé par Bertaux (1997) ou Desgagné et al. (2001) comme une forme de narration d'une histoire la plus à même de rendre compte du déroulement de l'action dans le temps, comme une étape significative dans une pratique. Ainsi, amener un acteur à se raconter dans une situation vécue lui permettrait de renouer avec les contraintes qu'il a prises en compte et les ressources qu'il a mobilisées pour affronter un problème. Considérée de la sorte, cette mise en discours fournit un moyen d'aborder par exemple l'usage fait par le praticien de son « répertoire d'actions » (Schön, 1983, 1994, 1996), une des dimensions d'intérêt évoquées précédemment. Les contraintes de la mise en mots de l'action en situation paraissent donc pouvoir être atténuées par une méthodologie de recherche adaptée à la particularité de l'évocation d'interventions préalablement vécues, telle qu'une démarche en collaboration qui permet au praticien de reconstruire l'action passée, dans le récit d'une pratique singulière.

Ainsi, s'appuyant sur ces fondements théoriques, nous inscrivons notre recherche dans une perspective qui tient l'acteur pour compétent, qui reconnaît la pertinence de ses savoirs pratiques développés grâce à la réflexivité qu'il engage au quotidien. Par ailleurs, nous retenons un moyen qui semble pertinent pour l'assister dans l'évocation d'expériences, alimentant en cela la compréhension des savoir-faire qu'il mobilise dans sa pratique professionnelle. C'est à partir de cette posture que nous avons élaboré un dispositif méthodologique centré sur la parole de l'acteur.

\section{De l'accueil et de l'interprétation du sens donné à la pratique}

Évoquer l'approche théorique qui a présidé à notre recherche a permis d'expliciter en quoi il est pertinent de donner la parole aux acteurs, parole conçue comme une voie privilégiée pour mieux appréhender les savoirs pratiques mobilisés dans le contexte 
d'une intervention. L'approche méthodologique employée, dont nous rapportons ici les éléments, s'inscrit en toute cohérence dans cette conception de l'acteur compétent susceptible de faire le récit d'une pratique singulière, lorsque sollicité.

C'est par le biais d'entretiens semi dirigés que nous avons invité des coachs à narrer l'une de leurs interventions de coaching en entreprise, en retenant le récit d'une pratique comme moyen d'expression, estimant que ce dispositif rencontre les objectifs décrits et relève les défis posés par notre investigation. Par la suite, notre approche inductive conduit à une construction de sens accordée aux données acquises et restituées sous forme de discours par un recours à l'analyse de contenu.

\section{Un entretien semi dirigé}

Comme l'énoncent par exemple De Ketele et Roegiers (1996) et Poupart (1997, 1993), l'entretien est l'outil privilégié par de nombreuses recherches de type qualitatif. Or, il existe différents types d'entretien, qualifiés d'individuel ou de collectif, de formel ou d'informel, d'ouvert ou de fermé, etc.

Pour notre recherche, nous avons employé l'entretien de type semi dirigé qui semblait le plus adéquat pour la poursuite de nos objectifs, dans la mesure où, tel que décrit par les auteurs, cette méthode d'investigation tend à faciliter la libre expression de l'acteur, tout en permettant au chercheur de cadrer le discours dans la perspective de la recherche; de plus, il serait susceptible de minimiser l'influence exercée par le chercheur et ses questions. Autrement dit, l'entretien semi dirigé prétend procurer un espace d'expression à l'interviewé dans la mesure où celui-ci laisse libre cours à sa pensée et à sa verbalisation, sans avoir à se restreindre en répondant à des questions précises. Cependant, précisons que le récit reste tout de même balisé par un cadre de narration, construit autour de l'objet de recherche et négocié dans le contrat de communication qui guide l'évocation. Bref, tel que l'avance Desgagné (2005), la narration de l'acteur soutenue par le chercheur en cours d'entretien représente une étape de «co-opération », qui revêt la forme d'une action conjointe. En effet, au cours de celle-ci, le praticien, détenteur de l'histoire, se raconte, tandis que le chercheur accompagne sa narration, en tentant de s'abstenir de juger ou d'approuver, garant du fil conducteur temporel et du focus choisis pour la reconstruction de cet événement en regard de la demande faite.

Ainsi, dans le cadre de notre recherche, nous nous attachons à définir quelques éléments en vue de préparer les rencontres, à élaborer des consignes inspirées des travaux de Desgagné et al. (2001) visant à baliser la narration. D'abord, nous déterminons les indications que nous souhaitons transmettre aux coachs afin qu'ils puissent s'y référer pour choisir l'intervention à narrer. Ainsi, nous leur demandons d'opter pour une situation précisément identifiée afin d'éviter un discours généraliste, un événement significatif qui fait sens pour lui dans sa pratique. Ce faisant, nous permettons aux coachs d'envisager la teneur de la rencontre à venir et de privilégier une expérience singulière et située. Ensuite, nous identifions les caractéristiques qui nous semblent pouvoir constituer un fil conducteur pour accompagner les coachs dans leur narration. À cet effet, nous choisissons un certain nombre de balises telles que la description des contextes pour mieux comprendre le sens qu'il accorde aux contraintes 
ou aux ressources qui l'influencent pour cadrer le discours dans la perspective de la recherche, ou encore telles que les gestes posés afin de centrer l'explicitation sur le « comment », c'est-à-dire les enchaînements des actions pour recomposer le récit dans toute sa complexité. Ainsi, nous retenons des caractéristiques qui amènent le praticien à décrire finement son engagement dans l'action.

C'est suite aux entretiens menés que des verbatims sont réalisés sur la base d'enregistrements produits au cours de ceux-ci. La transcription des rencontres est faite de façon quasi intégrale, en inscrivant nos interventions (reformulations, réitérations, interrogations), en notant certains éléments de contexte (les rires par exemple), en rapportant la plupart des répétitions ou onomatopées qui pouvaient émaillées un récit (par exemple, « oui, oui »), en faisant apparaître les hésitations (par exemple, « heu »). Même si ces différents éléments des récits ne sont pas nécessairement pris en compte lors de l'analyse, ils permettent de rendre présent le contexte de l'entretien lors de l'exploitation du verbatim.

Puis, au-delà de la première mise en mots réalisée par les acteurs, nous leur rendons l'entière propriété de leur récit en leur permettant d'amender la transcription que nous en avons faite; ils y apportent alors les ajustements qu'ils considèrent importants afin de rendre compte au mieux du sens qu'ils attribuent à leur pratique. Ainsi, ils ont, pour certains, voulu préciser leur dire ou encore donner des exemples complémentaires pour étayer leurs propos. Cette étape n'invalide pas le récit reconstitué mais s'inscrit dans la perspective de la reconnaissance de la compétence du praticien et de l'intérêt accordé à sa pratique, telle qu'il se la représente. Elle a pour objectif de permettre à l'acteur de reprendre possession de son récit, afin qu'il vérifie sa conformité au regard qu'il porte sur sa pratique. Par ailleurs, cette validation par l'acteur nous permet de répondre au critère de fidélité qui assure à l'acteur le respect du sens des propos tenus dans l'instant avec le sens de l'expérience vue de l'extérieur; cette position nouvelle pour l'acteur qui se découvre mis en mot peut l'amener à préciser, nuancer, établir des liens qui enrichissent le récit déposé. Ainsi, comme le soutient Desgagné (2005), le narrateur assume son expérience tant lorsqu'il se dit que lorsqu'il se lit. En ce sens, nous respectons le point de vue de l'acteur sur son expérience.

Bref, ces quelques éléments rapportés sur notre conception de l'entretien semi dirigé, mis en relation avec l'exploitation que nous en avons faite pour notre recherche, explicitent certains des principes mis en œuvre pour permettre aux coachs de se raconter dans une pratique singulière. Voyons maintenant le processus d'analyse de contenu qui nous a permis de donner du sens aux différents récits construits à partir des interventions narrées par les coachs.

\section{Une analyse des verbatims}

L'analyse de contenu est en relation avec différents types d'écrits et se décline selon différentes perspectives, telles les analyses de style, les études de procédés, les examens de structures, etc., comme en traitent différents ouvrages d'analyse qualitative (par exemple Bardin, 1986; L'Écuyer, 1987; Mucchielli, 1998; Paillé et Mucchielli, 2003). C'est une analyse de contenu dans le sens où la conçoit L'Écuyer (1987) que 
nous avons retenue pour construire notre compréhension des actions mises en œuvre par les coachs en fonction de thèmes clés que nous avons créés à partir des récits de pratique, et dont nous exposons ici les principales étapes.

L'Écuyer (1987) identifie plusieurs temps pour procéder à une analyse de contenu: la prise de connaissance du matériel à analyser, la délimitation d'unités de sens - recherche de formulations de sens proche - en relation avec l'objet de l'analyse, puis le regroupement en catégories génériques sur la base d'un dénominateur commun, mettant en évidence des caractéristiques identifiées par le chercheur. Les catégories dont il est question ici peuvent être plus ou moins préexistantes à l'analyse conduite. En effet, L'Écuyer (1987) rapporte trois modèles d'analyse : le premier, le «modèle ouvert », est construit autour de catégories qui sont créées à partir du contenu analysé; le second, le "modèle fermé », est plutôt conçu autour de catégories déterminées préalablement par le chercheur; finalement, le «modèle mixte » qui consiste en une combinaison des deux approches décrites précédemment. Pour notre recherche, nous avons opté pour le «modèle ouvert» tel que défini ci-dessus, car nous étions soucieuse de ne pas imposer des catégories pré-établies dans lesquelles «emboîter » les discours des coachs. Enfin, toujours selon L'Écuyer (1987), vient l'étape d'interprétation des données produites par ce travail de classification.

Ainsi, à partir de cette méthode d'analyse, nous exerçons dans un premier temps une lecture « flottante» des verbatims afin de nous remettre en contact avec le contexte du récit; ce faisant, nous renouons avec le fil de l'histoire, avec l'action relatée. Suite à cette première lecture, nous travaillons à dégager ce qui nous paraît être des actions effectuées par le coach. En effet, l'objet de la recherche visant à explorer entre autres le « répertoire d'actions » (Schön, 1983, 1987, 1996) mis en œuvre par ce dernier dans le cadre d'une intervention, nous orientons notre attention autour de la recherche de verbes d'action, d'intentions d'action, d'activités et de situations exprimées par le coach, en procédant à un repérage systématique dans notre corpus. Cela prend l'apparence de tout ou partie de phrases ou de paragraphes, découpés pour nous permettre d'y apposer un ou plusieurs mots-clés, en relation avec le sens que nous leur accordons en regard du récit.

Par la suite, procédant à une analyse de type « modèle ouvert », nous créons des thèmes en effectuant des regroupements de mots-clés présentant une certaine proximité de sens. À partir de là, il convient de procéder par itération, en fusionnant des thèmes redondants, en en scindant d'autres. Cette reconfiguration s'accompagne d'un travail sur le plan de la dénomination et de la définition du thème, visant à les expliciter plus avant. Pour ce faire, les retours aux récits sont fréquents afin de renouer avec l'événement narré dans sa globalité. Cette phase de documentation aide activement à lever les ambiguïtés latentes. Nous organisons ainsi l'ensemble des verbatims au sein de différents thèmes, considérant l'exhaustivité des propos recueillis.

Enfin, en regroupant les thèmes traitant de ce qui semble être un même domaine d'actions, nous constituons des catégories mutuellement exclusives qui permettent de donner un sens plus global à la démarche mise en œuvre par le coach. Une première version de la grille d'analyse est ainsi construite, et c'est la codification répétée des 
verbatims et l'ajustement subséquent de celle-ci qui nous amènent à la stabiliser. Finalement, l'ensemble des verbatims est codifié en fonction de la grille ainsi créée.

L'analyse de contenu conduite de la sorte nous permet donc de proposer une représentation de la pratique des coachs à partir du récit qu'ils en font, sur la base de perspectives qui reconnaissent la pertinence de leur point de vue sur celle-ci.

\section{Une réflexion critique sur l'approche employée}

Telle que nous venons de la présenter, l'approche mise en œuvre inscrit notre recherche dans une démarche inductive qui offre une place privilégiée à l'acteur en ce qu'elle s'appuie sur son expérience vécue, restituée par lui-même au cours d'entretiens semi dirigés. Pour autant, il est nécessaire de mettre à plat un certain nombre de choix qui teintent la production scientifique issue de notre démarche, notamment par rapport à nos inférences dans le processus ainsi que par rapport aux conceptions épistémologiques qui la sous-tendent. Dans cette optique, nous revenons sur le cadre théorique choisi ainsi que sur le dispositif méthodologique mis en œuvre afin de préciser la place respective que les coachs et nous-mêmes avons occupée dans le processus de recherche.

La reconnaissance des savoirs construits dans l'expérience amène à s'intéresser aux pratiques des acteurs et, qui plus est, à partir du point de vue qu'ils ont sur cellesci. En effet, la théorie sur le praticien réflexif développée par Schön $(1983,1994,1996)$ et retenue comme éclairage principal pour notre recherche se définit autour de concepts tels que « conversation réflexive » ou encore « répertoire d'actions », des construits qui renvoient à la réflexivité des acteurs sur leur expérience. Par conséquent, une meilleure compréhension des pratiques passe par un point de vue « intérieur », ce qui confère aux acteurs une position privilégiée pour en parler. C'est dans ce but que des chercheurs tels que nous accordent une place centrale à leurs discours, reconnaissant qu'ils sont les mieux placés pour nous parler de leur façon de «négocier les situations » qu'ils rencontrent, dirait Fourez (1999), des choix qu'ils ont arbitrés. Le postulat mis ainsi de l'avant consiste à reconnaître la pertinence du sens que l'acteur donne à sa pratique, et s'opérationnalise en prenant appui sur ce matériau pour construire une connaissance de la compétence mise en œuvre.

La reconstruction narrative de l'expérience empruntée dans notre recherche au récit de pratique prétend faciliter la reconstruction des savoirs pratiques des coachs. Pourtant, nous n'envisageons pas le récit de pratique comme un moyen d' « accès » à des souvenirs qui seraient l'exacte restitution d'un passé formellement enregistré. En fait, le récit, tel qu'évoqué par l'acteur, correspond à la commande qui lui en est faite. En d'autres termes, lors de sa narration, le coach fait émerger une forme qui répond aux critères de l'évocation, critères que nous lui avons d'ailleurs soumis. En effet, si nous avions modelé notre demande et nos consignes sur d'autres perspectives que l'action et son contexte, nous aurions obtenu une autre narration du même événement.

Nous convenons par ailleurs qu'en verbalisant son expérience, le coach en construit une restitution, retraçant l'expérience d'hier avec la compréhension qu'il en a aujourd'hui. Cette perspective s'inscrit dans un paradigme constructiviste qui, tel que conçu par Fourez, Engelbert-Lecomte et Mathy (1997), pose qu'il n'existe pas une 
réalité objective faisant office de référence absolue, ni d'informations «pures », mais bien des références relatives, des discours interprétés, car « toute information est déjà organisée par notre connaissance » (p.12). Autrement dit, et sans disqualifier le récit de pratique, il faut considérer, comme l'affirme Bertaux (1997), que l'évocation d'une pratique renvoie, non à des faits ${ }^{2}$, mais au sens que l'acteur donne à son action, et que ce sens est une « réalité » qu'il met en mots grâce à l'éclairage de toute ses expériences antérieures et postérieures à l'événement relaté.

De la même manière, il convient d'interroger la technique d'entretien. Il paraît intéressant d'envisager, à l'instar des propositions de Poupart (1997, 1993), une épistémologie qui définit le contenu d'un entretien comme le fruit d'une coconstruction issue de l'interaction entre les protagonistes de la rencontre. Selon cette perspective, le chercheur joue aussi un rôle actif dans cette phase de production des données, participant à leur élaboration par sa présence, par son interaction, par son attitude au cours de l'entretien. Quelles que puissent être ses conduites, il va influencer peu ou prou la constitution du récit en écoutant sans intervenir ou en relançant la narration.

Ce regard critique sur cette méthode ne déprécie pas l'entretien semi dirigé. Il permet d'être conscient, comme le mentionne Poupart (1997, 1993), que chacun des entretiens obtenus est unique au regard des échanges qui ont présidé à leur construction, et donc très situé dans l'espace et dans le temps. Il rend possible une certaine distanciation quant au statut des données issues d'une activité de coconstruction de sens autour d'une pratique entre le coach et nous-même.

Une distance critique face à l'activité interprétative à laquelle s'adonne le chercheur pour donner du sens aux verbatims issus des entretiens menés est également à prendre. En effet, les descriptions méthodologiques que nous avons faites laissent entrevoir que le chercheur a un rôle actif dans l'attribution de sens qu'il donne au récit qu'il analyse. Qu'il applique des catégories préconçues ou qu'il cherche à en dégager des verbatims, il crée une image, un modèle, à partir de sa compréhension de l'événement décrit, de sa position épistémologique par rapport aux données produites, et des cadres théoriques auxquels il se réfère. En effet, la construction interprétative posée par le chercheur sur son objet de recherche est guidée par l'ensemble de ces considérations. En ce sens, il intervient directement dans le traitement des données en leur accordant une signification au regard de son objet de recherche. C'est pourquoi les différents niveaux d'interprétation posés sur les données doivent s'accompagner d'un retour fréquent au récit lui-même, afin de limiter les inférences du chercheur qui peut avoir tendance à faire dire au verbatim ce qu'il souhaite y trouver.

Pourtant, l'analyse de contenu thématique n'est nullement une pratique de recherche à disqualifier, pour peu que soient explicités ses potentialités et ses limites. De fait, le statut des données doit être envisagé par rapport à l'objectif qu'elles permettent d'atteindre et circonscrit par des limites explicites. Ces précautions permettent de « resocialiser » la production scientifique, tel que le fait valoir Larochelle (2003), de montrer qu'elle n'est pas une activité au-dessus des chercheurs et de leurs projets respectifs. Et réinsérer des savoirs produits dans leur contexte de production 
permet de leur donner une dimension spatiale et temporelle, une histoire, qui ancre cette production tout en la relativisant. Ainsi, notre activité de reformulation et d'interprétation de l'expérience des coachs a été guidée par un projet précis, celui de reconstruire les savoirs pratiques de ces praticiens.

En conclusion, notre contribution au travers de cet article vise à faire valoir l'intérêt d'accorder une place centrale à l'acteur en recherche, à son point de vue, à la façon dont il rationalise son agir dans le cadre des limites liées à l'explicitation que nous avons évoquées et à en proposer une illustration au travers d'une recherche menée auprès de coachs. C'est ainsi que nous avons soutenu que donner à l'acteur une place dans la recherche sous-tend une certaine conception qui lui reconnaît sa capacité de réfléchir à son agir et à en rendre compte. Nous avons aussi relevé qu'il peut être nécessaire d'accompagner l'acteur dans son effort d'explicitation de son agir, afin de faciliter la mise en mots de pratiques tacites intégrées à ses pratiques. Et c'est là une des raisons qui amène à solliciter la participation de ce dernier: les savoirs dont peut témoigner son agir ne peuvent être mis en lumière par un seul regard " extérieur », leur reconstruction nécessitant le concours de cet acteur.

Cette perspective de recherche nous semble féconde en ce qu'elle donne un moyen de profiter de la multiplicité des expériences singulières de l'acteur, de la diversité de ses points de vue sur celle-ci, ce qui permet d'enrichir notre compréhension des pratiques mises en œuvre; elle permet également de construire des modèles interprétatifs en relation avec les contextes singuliers et ainsi produire des savoirs plus situés. À l'instar des chercheurs qui s'inscrivent dans la tradition sociologique de Chicago, elle nous permet de poser un regard autre, complémentaire, que seul un dispositif valorisant le point de vue de l'acteur sur son expérience peut faire émerger.

\section{Notes}

${ }^{1}$ Nous concevons que les propositions de Giddens donnant lieu à modèle d'acteur social constituent une perspective parmi d'autres représentations possibles, lesquelles peuvent faire appel à d'autres modèles traitant d'action sans mettre en scène d'acteur (par exemple, Parsons, 1937).

${ }^{2}$ Nous concevons, tel Fourez et al. (1997), que parler de « faits » revient à réifier un événement qui n'a de sens que dans un espace temporel, social et culturel, et ne rend compte que d'une interprétation possible d'une situation par un acteur qui y trouve du sens.

\section{Références}

Barbier, J.-M. (1996). Savoirs théoriques et savoirs d'action. Paris: Presses universitaires de France.

Bardin, L (1986). L'analyse de contenu. Paris: Presses universitaires de France.

Berry, M. (1996). Savoirs théoriques et gestion. Dans J.-M. Barbier (dir.), Savoirs théoriques et savoirs d'action (pp. 43-56). Paris: Presses universitaires de France.

Bertaux, D. (1997). Les récits de vie. Perspectives ethnosociologiques. Paris: Nathan Université. 
Blumer, H. (1937). Social psychology. In E.P. Schmidt (eds.), Man and society: A substantive introduction to the social science (pp. 144-198). New York (NY): Prentice-Hall.

Bourassa, B., Serre, F. et Ross, D. (1999). Apprendre de son expérience. Québec: Presses de l'Université du Québec.

Cyrulnik, B. (2003). Les sentiers de chèvres et l'autoroute ou le premier temps de l'éthique. Dans V. Duclert, et A. Chatriot (dir.), Quel avenir pour la recherche? Cinquante savants s'engagent. Paris: Flamarion.

de Ketele, J.-M. et Roegiers, X. (1996). Méthodologie du recueil d'informations: fondements des méthodes d'observations, de questionnaires, d'interviews et d'étude de documents. Bruxelles: De Boeck Université.

Desgagné, S. (2005). Récits exemplaires de pratiques enseignantes. Analyse typologique. Québec: Presses de l'Université du Québec.

Desgagné, S., Gervais, F. et Larouche, H. (2001). L'utilisation du récit de pratique: son potentiel pour le développement professionnel des enseignants et autres éducateurs du monde scolaire. Dans A. Beauchesne, S. Martineau et M. Tardif (dir.), La recherche en éducation et le développement de la pratique professionnelle en enseignement (pp. 203-223). Québec: Éditions du CRP.

Desgagné, S. (1994). À propos de la «discipline de classe»: analyse du savoir professionnel d'enseignantes et d'enseignants expérimentés du secondaire en situation de parrainer des débutants. Thèse de doctorat non publiée, Québec, Université Laval.

Filteau, O., Bourassa, B. et Leclerc, C. (2001). Clientèles en voie de développement requièrent accompagnateurs. Rapport de recherche sur la reconnaissance des savoirs d'expérience des intervenantes et des intervenants des services d'intégration professionnelle. Québec: CRIEVAT Laval.

Fourez, G. (1999). Compétences, contenus, capacités et autres casse-têtes. Forum, mai, 26-31.

Fourez, G., Engelbert-Lecomte, V. et Mathy, P. (1997). Nos savoirs sur nos savoirs. Un lexique d'épistémologie pour l'enseignement. Paris, Bruxelles: De Boeck Université.

Guignon, Sylvie (2006). Des savoirs pratiques construits à partir de récits d'intervention de coachs en entreprise. Mémoire de maîtrise non publié, Québec, Université Laval.

Giddens, A. (1987). La constitution de la société. Paris: Presses universitaires de France.

Laborde, D. (1998). Enquête sur l'improvisation. Dans M. De Fornel et L. Quéré (dir.), La logique des situations. Nouveaux regards sur l'écologie des activités sociales (pp. 261-299). Paris: Éditions de l'École des Hautes Études en Sciences Sociales. 
Larochelle, M. (2003). Rapport au savoir et socialisation à la cité scientifique. Dans S. Maury et M. Caillot (dir.), Rapport au savoir et didactique (pp. 51-79). Paris: Fabert.

L'Écuyer, R. (1987). L'analyse de contenu: notion et étapes. Dans J.-P. Deslauriers (dir.), Les méthodes de la recherche qualitative (pp. 49-65). Québec: Presses de l'Université du Québec.

Liljenstrand, A. M. (2003). A comparison of practices and approaches to coaching based on academic background. Unpublished thesis, California (CA), Faculty of California School of Organizational Studies and Alliant International University.

Mucchielli, R. (1998). L'analyse de contenu des documents et des communications. Paris: ESF.

Paillé, P. et Mucchielli, A. (2003). L'analyse qualitative en sciences humaines et sociales. Paris: Armand Colin.

Parsons, T. (1937). The structure of social action. New York : Mac Graw-Hill.

Pessin, A. (2004). Un sociologue en liberté. Lecture de Howard S. Becker. Québec: Presses de l'Université Laval.

Poupart, J. (1997). L'entretien de type qualitatif: considérations épistémologiques, théoriques et méthodologiques. Dans Poupart, Deslauriers, Groulx, Laperrière, Mayer et Pires. La recherche qualitative. Enjeux épistémologiques et méthodologiques. Montréal : Gaëtan Morin éditeur.

Poupart, J. (1993). Discours et débats autour de la scientificité des entretiens de recherche. Sociologie et sociétés, 25(2), 93-110.

St-Arnaud, Y. (1997). Le savoir, un objet perturbateur non identifié (OPNI) dans l'intervention. Dans C. Nelisse (dir.). L'intervention: les savoirs en action. (pp. 165-181). Paris: Presses universitaires de France.

Schön, D.A. (1983). The reflective practitioner. New York: Basic books.

Schön, D.A. (1994). Le praticien réflexif. À la recherche du savoir caché dans l'agir professionnel. Montréal: Les Éditions Logiques.

Schön, D.A. (1996). À la recherche d'une nouvelle épistémologie de la pratique et de ce qu'elle implique pour l'éducation des adultes. Dans J-M. Barbier (dir.), Savoirs théoriques et savoirs d'action (pp. 201-222). Paris: Presses universitaires de France.

Sylvie Guignon est doctorante en psychopédagogie à l'Université Laval. Détentrice d'une maîtrise en psychopédagogie de l'Université Laval et d'une maîtrise en informatique de gestion de l'Université de Rennes (France), ses travaux de recherche et ses publications puisent aux domaines du management, des savoirs pratiques, de l'interactionnisme symbolique ainsi qu'à la sociologie des professions. Elle y privilégie les méthodes qualitatives et les recherches participatives.

Joëlle Morrissette est doctorante en psychopédagogie à l'Université Laval. Ses travaux de recherches et ses publications croisent trois domaines: celui de l'évaluation des 
apprentissages, de l'interactionnisme symbolique ainsi que celui des savoirs pratiques. Ses champs d'intérêt professionnel s'orientent sur le plan théorique vers la sociologie et les perspectives interactionnistes, de même que l'épistémologie des sciences. Sur le plan méthodologique, ils s'orientent vers l'analyse de discours et vers les investigations collaboratives sur le terrain par le biais d'entretiens avec les enseignantes et les enseignants. 